RESEARCH ARTICLE

\title{
Effects of a Heart Rate Variability Biofeedback Intervention on Athletes Psychological Responses Following Injury: A Pilot Study
}

\author{
Scott Rollo ${ }^{1 *}$, Jill Tracey ${ }^{2}$ and Harry Prapavessis ${ }^{1}$ \\ ${ }^{1}$ Exercise and Health Psychology Laboratory, School of Kinesiology, The University of Western Ontario, \\ Canada \\ ${ }^{2}$ Department of Kinesiology \& Physical Education, Wilfrid Laurier University, Canada
}

*Corresponding author: Scott Rollo, Exercise and Health Psychology Laboratory, School of Kinesiology, Faculty of Health Sciences, The University of Western Ontario, London, N6A 5B9, Canada, E-mail: arollo@uwo.ca

\begin{abstract}
Context: In sport, the occurrence of an injury is almost inevitable and the rehabilitation process can take a toll on athletes' emotions and contribute to psychological distress.

Objective: To examine the effects of heart rate variability biofeedback (HRV BFB) training on pain catastrophizing and the psychological response variables of injured athletes. As a manipulation check, HRV BFB effects on athletes physiological indices including frequency-domain HRV and respiration rate were also assessed.

Design: A pilot randomized controlled trial.

Setting: University laboratory.

Participants: Twenty-eight athletes $\left(M_{\text {age }}=20.82, \mathrm{SD}=\right.$ 3.41 , Male $=19$ ) who sustained a moderate to severe musculoskeletal sports injury and underwent rehabilitation were randomized into HRV BFB experimental, placebo, or control conditions.

Intervention: Participants in the experimental condition received six 30-min HRV BFB training sessions over a 3-week period.

Main outcome measures: Psychological responses to sport injury, pain catastrophizing, and physiological indices were assessed at Baseline, Week 1, Week 2, and Week 3 (post-intervention).

Results: Significant training effects on respiration rate and low frequency HRV at rest were found in favour of the experimental group. For pain catastrophizing constructs and psychological responses to sport injury, group differences over time were non-significant; however, the effects were medium to large and consistently favoured the experimental group ( $\eta^{2}$ range $0.06-0.13$ ). Only isolation reached significance with a large effect in favour of the experimental group
\end{abstract}

group ( $p=0.024, \eta^{2}=0.17$ ). Significant, positive associations were found between change in respiration rate-rest and changes in rumination, magnification, devastation, restlessness, and isolation.

Conclusion: Preliminary evidence indicates that HRV BFB training may be a promising intervention to improve pain catastrophizing and the psychological responses of injured athletes throughout the rehabilitation process. A large, adequately powered randomized controlled trial is warranted.

\section{Keywords}

Biofeedback, Sport injury, Heart rate variability, Psychological rehabilitation

\section{Introduction}

Participation in sport is associated with the possibility of athletic injury. The 2013-2014 Statistics Canada Community Health Survey indicated $33.5 \%$ of injuries were a result of participation in sport and exercise [1]. Additionally, an estimated eight million sports injuries are treated annually in Europe [2] and over 3.3 million athletic related injuries are reported in the United States annually [3].

While the physical aspects of injury continue to be the focal point of injury research and rehabilitation, researchers have shown recovery from injury entails more than physical healing $[4,5]$. Cognitively, athletes need to understand the nature of the injury; emotionally, they must deal with adverse affect; and behaviorally, they must actively cope with their condition [4]. According 
to Wiese-Bjornstal, et al. integrated model of psychological responses to the sport injury and rehabilitation process, both pre- and post-injury factors, as well as numerous personal (e.g., injury history, severity, type) and situational (e.g., sport type, level of competition, playing status) variables influence an athlete's cognitive appraisal of the injury, which in turn, influences their emotional and behavioural responses to injury and subsequent recovery outcomes [6]. It has been shown that injury rehabilitation, particularly the initial phase of sustaining the injury, is associated with the greatest mood disturbance [7]. Tracey [7] reported athletes described a "roller coaster" of emotions, characterized by feelings of loss, decreased self-esteem, frustration, and anger, as they attempted to cope with their injury and subsequent rehabilitation. Johnston and Carroll [8] found that injured athletes reported feelings of shock, disbelief, guilt, jealousy, apathy, and fear following an injury and throughout rehabilitation. Other psychological responses to injury that have been cited in the literature include isolation, anxiety, depression, impatience, lack of confidence, and attempts to rationalize [5,7-9].

In addition to unfavorable emotions that may occur following injury, pain and discomfort can discourage athletes and prevent them from achieving their rehabilitation goals [10]. Research has shown that pain-related psychological variables can have a negative impact on recovery outcomes following musculoskeletal injury [11]. Pain catastrophizing has been defined as an exaggerated negative orientation towards actual or anticipated pain and is characterized by feelings of helplessness, active rumination and excessive magnification $[11,12]$. There is considerable research evidence that pain catastrophizing contributes to heightened pain intensity, pain-related disability, and psychological distress $[13,14]$. For example, in a sample of patients diagnosed with musculoskeletal pain receiving physical therapy, Bergbom, et al. found that higher levels of pain catastrophizing predicted lack of improvement and continued disability [15].

Due to the psychological challenges of injury underscored above, researchers have explored the implementation of psychological-based interventions to assist individuals in their rehabilitation such as goal setting [16], relaxation [17], stress inoculation training [18], modeling [19], and imagery [20]. All of these psychological-based skills have been shown to be associated with improved psychological responses and/or pain management among injured athletes. Despite the potential use of psychological-based interventions to assist injured athletes, less is known about combining psychological and physiological strategies such as Biofeedback (BFB) to assess potential application in rehabilitation. $\mathrm{Re}-$ search is warranted to explore beyond traditional psychological skills in rehabilitation and the incorporation of BFB has gained traction in recent years in some areas of sport psychology [21] and rehabilitation psychology
[22]. Theoretical models such as the biopsychosocial model assist in broadening the focus of injury by providing logical and reciprocal links between psychological and physiological influences [23].

\section{The use of heart rate variability biofeedback and related physiology}

The aim of BFB is to increase voluntary control over the physiological processes that are otherwise outside one's awareness, by using information about these in the form of an external signal or cue [24]. One modality of $B F B$ that is of particular interest is Heart Rate Variability Biofeedback (HRV BFB). Heart Rate Variability (HRV) refers to the beat-to-beat changes in the duration of $R R$ intervals (time between beats) in the Electrocardiogram (ECG) [25]. HRV has been suggested to be a reliable assessment of autonomic function, an index of cardiovascular adaptability, and indicative of autonomic or sympathovagal balance [26-28]. According to Appelhans and Luecken, high HRV reflects improved adaptability of physiological arousal, in addition to a greater capacity for regulated emotional responding [29]. Unfortunately, athletic injury and the subsequent rehabilitation period may have consequences on HRV. Olivier and colleagues examined HRV before and after a hospitalization period in a sample of amateur soccer players who had undergone knee surgery and found the hospitalization period led to an increase in resting heart rate, decrease in HRV (i.e., $7 \%$ reduction in mean $\mathrm{R}-\mathrm{R}$ interval, $66 \%$ reduction in total spectral power), and an overall disturbance of the autonomic nervous system [30].

HRV BFB training is designed to produce increases in HRV and to improve autonomic reactivity (For a review see Lagos, et al. 2008 [25]). HRV BFB training, through paced breathing at one's resonant frequency, produces resonance within the cardiovascular system, elicits High-Amplitude Heart Rate (HR) oscillations, and stimulates and exercises the baroreflexes, thereby improving modulation of autonomic activity $[25,31]$. In clinical populations, HRV BFB interventions have been implemented for a wide range of medical conditions and chronic illnesses as a means to improve upon autonomic dysfunction and with the goal of symptom reduction. Hallman, et al. [32] investigated the effects of HRV BFB on autonomic regulation and perceived health, pain, stress and disability in 24 subjects with stress- related chronic neck and shoulder pain. Findings demonstrated that those in the HRV BFB group achieved significantly greater increases in low-frequency HRV and health-related quality of life indices (i.e., vitality, bodily pain, and social functioning) across sessions compared to their control counterparts. In the sport realm, HRV BFB has been implemented for performance enhancement and emotional self-regulation [28]. Researchers have demonstrated its efficacy to improve psychomotor performance, cognitive and psychological states, and physiological functioning [28]. For example, Paul and 
Garg [28] examined the effectiveness of HRV BFB on the performance psychology and emotional self-regulation of anxious basketball players who competed at a university, state, and/or national level. Findings indicated that the HRV BFB group exhibited significantly greater improvements in anxiety, basketball performance skills (shooting, passing, dribbling), HRV (i.e., increases in total HRV and LF HRV), and respiration rate compared to those in the placebo and control groups. Specific to sport injury rehabilitation, Draper and Ballard [33] provided evidence for the effectiveness of Electromyographic (EMG) BFB to facilitate the recovery of quadriceps femoris muscle function following anterior cruciate ligament surgery. However, HRV BFB to reduce the emotional distress, while enhancing psychological skills (e.g. relaxation, coping) associated with optimal injury recovery outcomes has yet to be examined.

Given the genesis of the study to evaluate the efficacy of HRV biofeedback to improve athletes' psychological responses following an injury, the purpose of this pilot study was to examine the effects of HRV BFB training on pain catastrophizing and the psychological response variables of injured athletes. As a manipulation check, HRV BFB effects on athletes' physiological indices including HRV and respiration rate were also assessed. A pilot study was executed as it is a "small scale investigation designed to test the feasibility of methods and procedures for later use on a large full-scale study or to search for possible effects and associations that may be worth following up in a subsequent larger study" (p.1) [34]. Three hypotheses were generated based on preceding literature and our study objectives. First, injured athletes receiving the HRV BFB intervention would demonstrate positive changes in frequency-domain measures of HRV (i.e., total HRV, low-frequency HRV, high-frequency HRV) and respiration rate compared to those in the placebo and control groups. Second, injured athletes in the HRV BFB intervention group would report greater increases in the psychological response of reorganization; greater reductions in psychological responses of devastation, feeling cheated, restlessness, and isolation; and greater declines in pain catastrophizing variables over time compared to those in the placebo and control groups. Finally, it was hypothesized that changes in physiological indices associated with BFB training would be related to changes in pain catastrophizing and psychological response variables of injured athletes.

\section{Methods}

\section{Design}

This pilot investigation was a single-blinded, repeated measure, randomized controlled trial to assess group differences over time. A 3 (Group: HRV BFB experimental group, HRV BFB placebo group, control group; experimental conditions) $\times 4$ (Time: Baseline, Week 1, Week 2, Week 3; assessments across time) mixed model de- sign was utilized. Participants were blinded to group allocation and were unaware of the existence of the other conditions.

\section{Participants}

Participants had sustained a moderate to severe musculoskeletal sports injury. A moderate injury requires 8-28 days away from training and competition, and a severe injury requires up to 428 days or more away from training and competition [35]. At time of recruitment, participants were at varying stages of their rehabilitation. Additional inclusion criteria included (a) Participants to be at least 16-years-old, (b) Injuries to have occurred during participation in sport, (c) Participants to be out of practice and/or competition for the sport in which the injury occurred, (d) Participants to be undergoing rehabilitation for the injury (e.g., physiotherapy, massage therapy, athletic therapy), and (e) Participants to be involved in sport at a competitive level (e.g., elite club, university, provincial, national) and/or to be engaging in sport in a competitive environment (e.g., adult competitive league, training for specific events, competitions, etc.). Exclusion criteria included anyone with known medical disorders and/ or heart abnormalities (e.g., cardiac arrhythmia) that would impede them from performing the biofeedback procedures; those receiving formal training in any form of relaxation, biofeedback, or breathing technique; and/ or those receiving another psychological intervention. Participant characteristics are provided in Table 1.

\section{Intervention}

HRV BFB experimental group: Participants in the HRV BFB experimental group received six HRV BFB training sessions over a three-week period, each roughly $30 \mathrm{~min}$ in duration. The protocol designed by Lehrer, Vaschillo, and Vaschillo [36] for HRV BFB training, which has been used extensively in research, was implemented (see Lehrer, et al. [31] for details of training). The training sessions involved instruction in paced breathing at one's resonant frequency emphasizing maximizing HRV. Participants were asked to sit with their eyes closed in a semi-reclined position for 5 min while attempting to relax and breathe normally. Participants were then provided with 20 min of HRV BFB training.

In the first session, the participants were taught to breathe at their resonant frequency, determined by asking the participant to breathe at variable respiratory rates for 2 min each $(6.5,6.0,5.5,5.0,4.5$ breaths per minute). A pacing stimulus was provided on the computer screen to aid in this task. The resonant frequency was identified as the respiratory frequency yielding the highest low-frequency HRV on the moving Fourier power spectrum displayed by the AD Instruments LabChart ${ }^{\circledR}$ 7.0 software. In subsequent sessions, participants were trained how to breathe using pursed lips and abdominal breathing techniques with prolonged exhalation in or- 
Table 1: Participant Demographic Information Based on Group.

\begin{tabular}{|c|c|c|c|c|}
\hline \multicolumn{2}{|l|}{ Variable } & \multicolumn{3}{|l|}{ Group } \\
\hline & & $\begin{array}{l}\text { Experimental } \\
(n=9)\end{array}$ & $\begin{array}{l}\text { Placebo } \\
(n=9)\end{array}$ & $\begin{array}{l}\text { Control } \\
(n=10)\end{array}$ \\
\hline Age (Years) & & 22.0 years & 21.0 years & 19.6 years \\
\hline$M(S D)$ & & (5.5) & (1.5) & (1.7) \\
\hline \multirow[t]{4}{*}{$\operatorname{Sex}(M / F)$} & & 5 Females & 1 Female & 3 Females \\
\hline & & $(55.6 \%)$ & $(11.1 \%)$ & $(30 \%)$ \\
\hline & & 4 Males & 8 Males & 7 Males \\
\hline & & $(44.4 \%)$ & $(88.9 \%)$ & $(70 \%)$ \\
\hline \multirow[t]{5}{*}{ Injury History } & No & $1(11.1 \%)$ & $1(11.1 \%)$ & $4(40 \%)$ \\
\hline & & $3(33.3 \%)$ & $1(11.1 \%)$ & $1(10 \%)$ \\
\hline & & $1(11.1 \%)$ & $1(11.1 \%)$ & $2(20 \%)$ \\
\hline & & $2(22.2 \%)$ & $2(22.2 \%)$ & $2(20 \%)$ \\
\hline & & $2(22.2 \%)$ & $4(44.4 \%)$ & $1(10 \%)$ \\
\hline \multirow[t]{8}{*}{ Sport } & Soccer & $3(33.3 \%)$ & $2(22.2 \%)$ & $3(30 \%)$ \\
\hline & Football & & $3(33.3 \%)$ & $3(30 \%)$ \\
\hline & Hockey & $2(22.2 \%)$ & $3(33.3 \%)$ & $1(10 \%)$ \\
\hline & Rugby & $1(11.1 \%)$ & $1(11.1 \%)$ & $2(20 \%)$ \\
\hline & Baseball & & & $1(10 \%)$ \\
\hline & Basketball & $1(11.1 \%)$ & & \\
\hline & Cheerleading & $1(11.1 \%)$ & & \\
\hline & Ultra-running & $1(11.1 \%)$ & & \\
\hline \multirow[t]{4}{*}{ Competition Level } & Varsity/university & $7(77.8 \%)$ & $9(100 \%)$ & $9(90 \%)$ \\
\hline & Competitive & $1(11.1 \%)$ & & $1(10 \%)$ \\
\hline & Recreational & & & \\
\hline & Other & $1(11.1 \%)$ & & \\
\hline \multirow[t]{5}{*}{ Injury Type } & Knee & $4(44.4 \%)$ & $3(33.3 \%)$ & $5(50 \%)$ \\
\hline & Нip & $1(11.1 \%)$ & $4(44.4 \%)$ & \\
\hline & Ankle & $2(22.2 \%)$ & & $3(30 \%)$ \\
\hline & Shoulder & & $1(11.1 \%)$ & $1(10 \%)$ \\
\hline & Other & $2(22.2 \%)$ & $1(11.1 \%)$ & $1(10 \%)$ \\
\hline \multirow[t]{3}{*}{ Time Since Injury Onset } & Less than week & $1(11.1 \%)$ & $1(11.1 \%)$ & \\
\hline & More than week, less than month & $5(55.6 \%)$ & $5(55.6 \%)$ & $3(30 \%)$ \\
\hline & More than month & $3(33.3 \%)$ & $3(33.3 \%)$ & $7(70 \%)$ \\
\hline Perceived Pain (0-10) M (SD) & & $3.22(1.92)$ & $2.22(2.39)$ & $3.00(2.35)$ \\
\hline
\end{tabular}

der to elicit high amplitude oscillations in HR at their resonant frequency. Participants were provided with BFB (i.e., physiological data) in numerous forms. Utilizing the physiological data displayed (e.g., beat-to-beat HR display, respiratory activity, and respiratory pacer), participants were instructed to increase the amplitude of $\mathrm{HR}$ oscillations that occur in phase with respiration, with the goal to teach participants to self-regulate by increasing their awareness and control over their breathing and HR. Participants were also encouraged to practice breathing at their resonant frequency for 20 min daily outside of training (e.g., metronome, pacer application available for Smartphones).

HRV BFB placebo group: Participants received six BFB sessions over the three-week duration, each roughly $30 \mathrm{~min}$ in length; however, no instruction in paced breathing or techniques to maximize HRV was provided. Participants were asked to sit with their eyes closed in a semi-reclined position for $5 \mathrm{~min}$ while attempting to relax and breathe normally; then, provided with $20 \mathrm{~min}$ of BFB. Participants still received the same on-screen physiological information through $B F B$, however, no ad- ditional information, other than to relax and continue their normal breathing pattern was given. Participants were also encouraged to practice relaxing daily outside of the BFB sessions.

Control group: Participants in the control group received no training of any kind over the duration of the study and were only asked to attend data collection sessions. At study completion these participants were offered training in BFB and provided resources for BFB.

\section{Manipulation Check Measures}

Physiological indices: Participants completed an assessment for measurement of frequency-domain HRV and respiration rate. Participants sat in a semi-reclined position while remaining comfortable and relaxed. Data were recorded during two 10 min tasks: Task A - Rest Period and Task B - Paced Breathing Exercise. For Task A, participants were instructed to relax as much as possible and breathe normally. Following this, participants completed Task B, a paced breathing exercise in which they were instructed to utilize the biofeedback information provided on the computer screen and match the 
rhythm of their breathing to a slowly oscillating respiratory pacer, set at six breaths per minute. A spectral power analysis was administered over each of the tasks by means of a Fast Fourier transformation. For both tasks, HRV frequency-domain values and average respiration rate (breaths per minute) were measured. Total power (Total HRV), and power values for two frequency bands, low-frequency (0.04-0.15 Hz; LF HRV) and high-frequency (0.15-0.4 Hz; HF HRV) were assessed. Given previous research and current study objectives, LF HRV and respiration rate during Task A (rest) were considered the most salient physiological outcomes of interest.

\section{Primary outcome measures}

Psychological responses to sport injury: Psychological responses were assessed using the 19-item Psychological Responses to Sport Injury Inventory (PRSII) [37]. The PRSII has five subscales; devastation (i.e., reflects feelings of intense shock and emptiness), feeling cheated (i.e., reflects bitterness at being injured), restlessness (i.e., characterized by feelings of anxiety, guilt, and hostility), reorganization (i.e., represents increased confidence and a sense of psychological recovery), and isolation (i.e., represents feelings of isolation). Items are measured on a 5-point Likert scale anchored at 5 (strongly agree) and 1 (strongly disagree). Each subscale score (with the exception of reorganization) ranges from a low of 4 to a high of 20 . This equates to a low score of 3 and a high score of 15. Evans, et al. [37] provided evidence of content and predictive validity. Cronbach's alpha coefficients averaged over the time phases of injury were 0.82 for devastation, 0.75 for reorganization, and 0.77 for feeling cheated, 0.85 for restlessness, and 0.72 for isolation.

Pain catastrophizing: The Pain Catastrophizing Scale (PCS) was used to assess the degree to which various thoughts and feelings surrounding pain are experienced [12]. It captures the extent of a patient's negative or exaggerated orientation to pain and it addresses primary constructs of rumination, magnification, and helplessness [12]. The PCS is a 13-item questionnaire with items being ranked using a 5-point scale, ranging from 0 (not at all) to 4 (all the time) and scored by summing responses for all 13 questions. The PCS has demonstrated adequate to excellent internal consistency (rumination = 0.87 , magnification $=0.66$, and helplessness $=0.78$ ).

\section{Equipment and Technical Procedures}

All HRV BFB training and assessments were administered using an AD Instruments Power Lab 26T biofeedback unit with LabChart ${ }^{\circledR} 7.0$ software and the HRV module add-on ( $A D$ Instruments). Respiratory patterns were collected using a respiration-monitoring belt with sensors placed around the participant's upper abdomen. HR and cardiac rhythm were measured using a finger-pulse transducer and ECG sensors, connected to participants via three electrodes (Kendall ${ }^{\mathrm{TM}} 130$ Foam Electrodes, Conductive Adhesive Hydrogel) placed on the medial aspect of the participants' forearms. The raw ECG signal, instantaneous HR (i.e., pulse \& ECG; beats per minute), respiratory sinus arrhythmia graph, respiration rate and a respiratory pacer (for Task $B$ and/or HRV BFB experimental group training only) were presented to the participants.

\section{General procedures}

Ethics approval was granted by the Research Ethics Board at the authors' institution (\#3670) and consent was obtained from all participants. Participants were recruited from varsity athletics programs at a medium-sized university via the athletic therapy clinic and from three clinics specializing in athletic injury rehabilitation. Eligibility was determined via the recruitment letter and an initial e-mail screen, and if necessary, further, by the demographics questionnaire and one screening visit (Baseline). Once deemed eligible, participants were randomly assigned to one of three groups: (a) HRV BFB experimental group (i.e., full protocol with abdominal breathing through pursed lips and prolonged exhalation; $n=9$ ); (b) HRV BFB placebo group $(n=9)$; or (c) Control group $(n=10)$. At the baseline (pre-intervention) visit, eligible participants were thoroughly informed regarding study details, and provided with an informed consent (applicable to their assigned group) to complete. The participant then completed a demographic questionnaire, followed by baseline self-report measures of psychological outcome variables. Participants were then introduced to the setting, equipment, and basic procedures of biofeedback. Finally, an assessment was administered to obtain participants' baseline physiological data (i.e., HRV and respiration measures).

In addition to the baseline assessment, psychological outcomes and the physiological indices were measured at Week 1 (i.e., Day eight), Week 2 (i.e., Day 15), and Week 3 (i.e., Post-intervention, Day 22). All BFB training and data collection sessions were conducted in the physiology laboratory at the authors' institution, with an ambient room temperature $\left(20^{\circ} \mathrm{C}-25^{\circ} \mathrm{C}\right)$ and sound attenuation.

\section{Statistical Analyses}

Missing data: Prior to data analysis, all missing data points were coded by imputing a random numerical value (e.g., 2.00). The statistical analyses performed on the data allowed for missing observations under a missing at random assumption. For any missing values the participant was subsequently excluded from the analysis for that variable. After computing outcome variables, reliability analyses were run and Cronbach's alphas were calculated.

Group equivalency: Separate ANOVAs were conducted to determine if any baseline differences existed among treatment groups on demographic, psychological, and physiological variables. For any demographic variables where baseline group differences were found, separate ANOVAs were conducted with these as the 
independent factor to examine effects on baseline psychological variables. If significant or moderate to large non-significant effects were found for any of the demographic variables that also showed an association with any of the psychological variables, they were treated as covariates for the subsequent primary analyses. If baseline treatment group differences were found for any of the physiological or psychological variables, they were treated as covariates for subsequent manipulation check and primary analyses, respectively.

Manipulation check: Eight 3 (Group: HRV BFB experimental group, HRV BFB placebo group, control group) $\times 4$ (Time: Baseline, week 1, week 2, week 3) repeated measure ANOVAS\ANCOVAS were used to determine whether group differences could be seen across time for physiological variables during both Task A - Rest and Task B - Paced. Simple contrasts with Baseline as the reference category were run for significant Group by Time interaction effects. Changes in these physiological variables associated with biofeedback training served as the manipulation check.

Primary analyses: Separate $3 \times 4$ repeated measure ANOVAS $\backslash A N C O V A S$ were conducted for each psychological variable to identify condition by time interaction effects. For these repeated measure analyses the Mauchly's test of sphericity was confirmed by a non-significant statistic ( $p>0.05)$. If non-significant, within-subjects effects were reported; otherwise, violations to this assumption $(p<0.05)$ were accounted for by reporting multivariate statistics [37].

Relationship among the variables: Absolute change scores for the physiological variables were calculated by subtracting the Baseline scores from those collected at Week 3 (for respiration rate, change score was calculated by subtracting Week 3 scores from Baseline

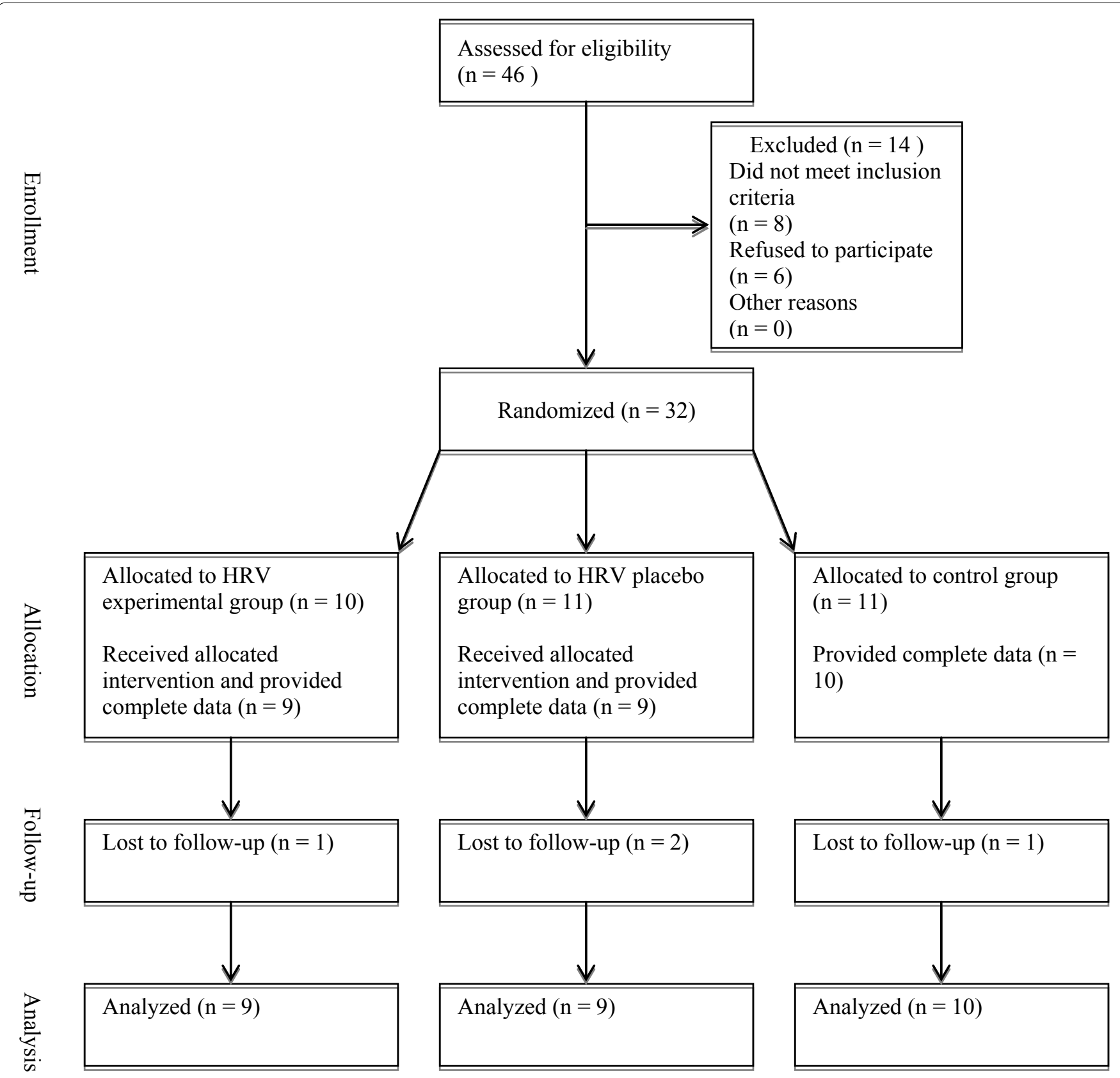

Figure 1: Flow diagram of participant progress through the phases of the randomized controlled trial pilot investigation. 
scores). Absolute change scores for psychological response to sport injury and pain catastrophizing variables were calculated by subtracting the Week 3 scores from those collected at Baseline (for psychological response subscale of reorganization, change score was calculated by subtracting Baseline scores from Week 3 scores). Pearson's product moment correlation analyses were then conducted to examine the relationships between change in physiological variables and changes in psychological variables (i.e., psychological responses to sport injury, pain catastrophizing).

Power and statistical analysis: No previous research exists to inform a sample power calculation for psychological response to rehabilitation for injury following a HRV intervention. Statistical analyses were performed using the Statistical Package for Social Sciences (SPSS) version 24.0. AP value $<0.05$ was regarded as significant for all statistical tests and a partial-eta squared $\left(\eta^{2}\right)$ of $0.01,0.06$, and 0.14 represented small, medium, and large effect sizes, respectively [38].

\section{Results}

Twenty-eight athletes recruited from athletic therapy and sports medicine clinics met eligibility criteria and agreed to participate (see Figure 1). Participants ranged in age from 18 to 36 years $\left(M_{\text {age }}=20.82, \mathrm{SD}=\right.$ 3.41) and consisted of 19 males and 9 females. No missing values were present for psychological response or pain catastrophizing variables; however, due to missed attendance $(n=1)$ and technical error $(n=2)$, participants were excluded from analyses for the physiological variables. For Task A - Rest Period variables, one participant $(n=1)$ was excluded from the analyses $(N=27)$. For Task B - Paced Breathing Exercise variables, three participants $(n=3)$ were excluded from the analyses $(N=$ 25). For this investigation, internal consistencies for the psychological response to sport injury subscales ranged across time points from $\alpha=0.82-0.92$ for devastation, $\alpha$ = 0.66-0.89 for reorganization, $\alpha=0.72-0.91$ for feeling cheated, $\alpha=0.62-0.93$ for restlessness, and $\alpha=0.73$ 0.86 for isolation. For pain catastrophizing subscales, the internal consistency for rumination was $\alpha=0.86$ at baseline, and 0.89, 0.89, 0.92 at Week 1, Week 2, and Week 3 respectively. The internal consistency for magnification was $\alpha=0.73$ at baseline, and $0.81,0.72,0.84$ at each subsequent time point. The internal consistency for helplessness was $\alpha=0.80$ at baseline, and $0.86,0.87$, 0.94 at each subsequent time point.

\section{Group Equivalency}

Baseline characteristics for the HRV experimental, HRV placebo, and control groups are shown in Table 1. There were no significant group differences for the demographic variables of age, $F(2,25)=1.209, p=0.315, \eta^{2}=0.088$, gender, $F(2,25)=2.149, p=0.138, \eta^{2}=0.147$, time since injury, $F(2,25)=1.991, p=0.158, \eta^{2}=0.137$, or injury history, $F(2,25)=1.760, p=0.193, \eta^{2}=0.123$. There were also no significant group differences with regards to perceived pain levels at baseline, $F(2,26)=0.500, p=0.613$, $\eta^{2}=0.040$. A significant effect of injury history (i.e., $0,1,2$, $3,4)$ was found on baseline pain catastrophizing variables, magnification, $F(4,23)=2.938, p=0.042, \eta^{2}=0.338$, and helplessness, $F(4,23)=3.677, p=0.019, \eta^{2}=0.39$, and psychological response to injury variables, devastation, $F$ $(4,23)=4.934, p=0.005, \eta^{2}=0.462$, feeling cheated, $F(4$, 23) $=3.379, p=0.026, \eta^{2}=0.37$, and restlessness, $F(4,23)=$ $4.419, p=0.009, \eta^{2}=0.435$, indicating those with two previous injuries consistently reported higher baseline scores. A significant main effect of gender was also found for the pain catastrophizing variable of rumination, $F(1,26)=$ 5.005, $p=0.034, \eta^{2}=0.161$, and psychological response variables of devastation, $F(1,26)=4.748, p=0.039, \eta^{2}=$ 0.154 , and feeling cheated, $F(1,26)=8.952, p=0.006, \eta^{2}=$ 0.256 . Females reported higher scores for rumination, devastation, and feeling cheated than males at baseline. Given the large, albeit non-significant effect indicating group differences for gender and injury history, and the fact that these were both significantly related with several primary psychological outcomes at baseline; it was deemed necessary to treat gender and injury history as covariates in subsequent primary analyses. With regards to physiological and psychological variables, there were no significant differences between the groups at baseline, with the exception of the psychological responses of devastation, $F(2$, $25)=4.071, p=0.029, \eta^{2}=0.246$, and reorganization, $F(2$, $25)=3.573, p=0.043, \eta^{2}=0.222$. Hence, baseline scores for these two constructs were treated as covariates in subsequent primary analyses.

\section{Manipulation Check}

Table 2 contains the group means and standard deviations for the physiological indices of respiration rate, LF HRV, HF HRV, and total HRV during both tasks at Baseline, Week 1, Week 2, and Week 3.

Respiration rate - rest: There was a large significant Group by Time interaction effect, $F(6,72)=7.368$, $p<$ $0.001, \eta^{2}=0.380$, indicating rates of breathing at the different time phases differed between groups. Decreases in mean respiration rate during Task $A$ - rest period from Baseline to Week 1, Week 2, and Week 3 for the experimental group were significantly greater than for either the placebo or control groups.

LF HRV - rest: There was a large significant Group by Time interaction effect found, $F(6,72)=2.72, p=0.019$, $\eta^{2}=0.185$. Increases in LF HRV - rest from Baseline to Week 1 and Baseline to Week 2 for the experimental group was significantly greater than for either the placebo or control groups.

HF HRV - rest: There was a medium non-significant Group by Time interaction effect found, $F(6,72)=0.986$, $p=0.441, \eta^{2}=0.076$. The groups essentially remained the same across time.

Total HRV - rest: There was a medium to large 
Table 2: Descriptive Statistics for Physiological Outcomes at Baseline, Week 1, Week 2, and Week 3.

\begin{tabular}{|c|c|c|c|c|c|c|}
\hline \multirow{3}{*}{ Variable } & \multirow{3}{*}{ Group } & \multirow{3}{*}{$\mathbf{n}$} & \multicolumn{4}{|l|}{ Assessment } \\
\hline & & & Baseline & Week 1 & Week 2 & Week 3 \\
\hline & & & $M(S D)$ & $M(S D)$ & $M(S D)$ & $M(S D)$ \\
\hline \multirow[t]{4}{*}{ Respiration Rate - Rest } & Experimental & 9 & 12.59 & 6.61 & 6.09 & 6.67 \\
\hline & & & $(2.53)$ & (1.04) & $(1.14)$ & (1.32) \\
\hline & Placebo & 9 & $12.50(4.71)$ & $11.35(3.08)$ & $12.51(5.25)$ & $12.14(4.71)$ \\
\hline & Control & 9 & $13.82(3.41)$ & $12.84(3.98)$ & $12.55(3.87)$ & $12.53(3.68)$ \\
\hline \multirow[t]{3}{*}{ LF HRV - Rest } & Experimental & 9 & $\begin{array}{l}1917.23 \\
(2192.45)\end{array}$ & $\begin{array}{l}10535.90 \\
(7673.33)\end{array}$ & $\begin{array}{l}7764.45 \\
(4229.39)\end{array}$ & $\begin{array}{l}6030.91 \\
(4375.39)\end{array}$ \\
\hline & Placebo & 9 & $\begin{array}{l}4046.28 \\
(5943.67)\end{array}$ & $\begin{array}{l}5436.61 \\
(8051.19)\end{array}$ & $\begin{array}{l}3258.58 \\
(4724.04)\end{array}$ & $\begin{array}{l}4819.40 \\
(6141.06)\end{array}$ \\
\hline & Control & 9 & $\begin{array}{l}2035.99 \\
(1776.63)\end{array}$ & $\begin{array}{l}3080.32 \\
(4483.46)\end{array}$ & $\begin{array}{l}2913.88 \\
(3880.51)\end{array}$ & $\begin{array}{l}2758.06 \\
(4302.20)\end{array}$ \\
\hline \multirow[t]{3}{*}{ HF HRV - Rest } & Experimental & 9 & $\begin{array}{l}1534.71 \\
(1537.12)\end{array}$ & $\begin{array}{l}1149.07 \\
(1102.91)\end{array}$ & $\begin{array}{l}1461.31 \\
(2202.09)\end{array}$ & $\begin{array}{l}772.17 \\
(1147.13)\end{array}$ \\
\hline & Placebo & 9 & $\begin{array}{l}2521.14 \\
(2827.32)\end{array}$ & $\begin{array}{l}4026.19 \\
(5632.19)\end{array}$ & $\begin{array}{l}1739.16 \\
(1842.60)\end{array}$ & $\begin{array}{l}1989.98 \\
(1331.68)\end{array}$ \\
\hline & Control & 9 & $\begin{array}{l}1545.27 \\
(2748.84)\end{array}$ & $\begin{array}{l}1357.18 \\
(992.79)\end{array}$ & $\begin{array}{l}1497.74 \\
(944.34)\end{array}$ & $\begin{array}{l}926.04 \\
(567.80)\end{array}$ \\
\hline \multirow[t]{3}{*}{ Total HRV - Rest } & Experimental & 9 & $\begin{array}{l}4373.99 \\
(4003.15)\end{array}$ & $\begin{array}{l}12991.54 \\
(8815.61)\end{array}$ & $\begin{array}{l}11173.84 \\
(8130.79)\end{array}$ & $\begin{array}{l}8094.16 \\
(5525.49)\end{array}$ \\
\hline & Placebo & 9 & $\begin{array}{l}8581.49 \\
(6289.67)\end{array}$ & $\begin{array}{l}12917.80 \\
(13493.82)\end{array}$ & $\begin{array}{l}7504.58 \\
(6385.24)\end{array}$ & $\begin{array}{l}9091.39 \\
(6749.95)\end{array}$ \\
\hline & Control & 9 & $\begin{array}{l}5504.22 \\
(5760.63)\end{array}$ & $\begin{array}{l}6313.67 \\
(5130.24)\end{array}$ & $\begin{array}{l}7067.98 \\
(4202.07)\end{array}$ & $\begin{array}{l}5842.37 \\
(4649.94)\end{array}$ \\
\hline \multirow[t]{6}{*}{ Respiration Rate - Paced } & Experimental & 9 & 7.61 & 6.65 & 6.81 & 6.61 \\
\hline & & & $(2.08)$ & $(0.75)$ & $(0.93)$ & $(0.99)$ \\
\hline & Placebo & 9 & 7.25 & 7.09 & 6.99 & 7.14 \\
\hline & & & $(1.45)$ & $(1.00)$ & $(1.50)$ & (1.69) \\
\hline & Control & 8 & 7.73 & 7.03 & 6.76 & 7.22 \\
\hline & & & $(2.12)$ & (1.05) & $(0.76)$ & (1.54) \\
\hline \multirow[t]{3}{*}{ LF HRV - Paced } & Experimental & 9 & $\begin{array}{l}7518.97 \\
(3724.06)\end{array}$ & $\begin{array}{l}7305.14 \\
(4705.26)\end{array}$ & $\begin{array}{l}7591.59 \\
(4193.89)\end{array}$ & $\begin{array}{l}4636.20 \\
(2442.96)\end{array}$ \\
\hline & Placebo & 9 & $\begin{array}{l}9654.77 \\
(6375.36)\end{array}$ & $\begin{array}{l}13409.81 \\
(9488.48)\end{array}$ & $\begin{array}{l}9980.31 \\
(7171.43)\end{array}$ & $\begin{array}{l}10686.00 \\
(7108.84)\end{array}$ \\
\hline & Control & 7 & $\begin{array}{l}8292.53 \\
(5144.29)\end{array}$ & $\begin{array}{l}11556.51 \\
(8587.64)\end{array}$ & $\begin{array}{l}12121.95 \\
(7663.42)\end{array}$ & $\begin{array}{l}8374.81 \\
(6366.17)\end{array}$ \\
\hline \multirow[t]{3}{*}{ HF HRV - Paced } & Experimental & 9 & $\begin{array}{l}831.07 \\
(639.84)\end{array}$ & $\begin{array}{l}1362.45 \\
(1479.65)\end{array}$ & $\begin{array}{l}988.65 \\
(906.11)\end{array}$ & $\begin{array}{l}763.60 \\
(620.53)\end{array}$ \\
\hline & Placebo & 9 & $\begin{array}{l}1601.82 \\
(1243.66)\end{array}$ & $\begin{array}{l}2239.27 \\
(1923.10)\end{array}$ & $\begin{array}{l}1708.43 \\
(1160.03)\end{array}$ & $\begin{array}{l}1252.35 \\
(1059.45)\end{array}$ \\
\hline & Control & 7 & $\begin{array}{l}1506.44 \\
(1527.77)\end{array}$ & $\begin{array}{l}2362.43 \\
(1609.57)\end{array}$ & $\begin{array}{l}2175.48 \\
(1843.50)\end{array}$ & $\begin{array}{l}1464.51 \\
(1077.33)\end{array}$ \\
\hline \multirow[t]{3}{*}{ Total HRV - Paced } & Experimental & 9 & $\begin{array}{l}9893.85 \\
(5071.20)\end{array}$ & $\begin{array}{l}11662.33 \\
(7573.81)\end{array}$ & $\begin{array}{l}10329.41 \\
(5394.35)\end{array}$ & $\begin{array}{l}6766.24 \\
(3382.51)\end{array}$ \\
\hline & Placebo & 9 & $\begin{array}{l}15138.19 \\
(12525.72)\end{array}$ & $\begin{array}{l}18831.65 \\
(13318.33)\end{array}$ & $\begin{array}{l}14834.00 \\
(9680.58)\end{array}$ & $\begin{array}{l}14628.40 \\
(7974.81)\end{array}$ \\
\hline & Control & 7 & $\begin{array}{l}11197.02 \\
(7158.39)\end{array}$ & $\begin{array}{l}16399.34 \\
(11104.88)\end{array}$ & $\begin{array}{l}16872.11 \\
(11166.32)\end{array}$ & $\begin{array}{l}11639.24 \\
(8088.80)\end{array}$ \\
\hline
\end{tabular}

Note: LF HRV = Low Frequency; measured in $\mathrm{ms}^{2} / \mathrm{Hz}$; HRV in frequency band of 0.04-0.15 Hz. HF HRV = High Frequency; measured in $\mathrm{ms}^{2} / \mathrm{Hz} ; \mathrm{HRV}$ in frequency band of $0.15-0.4 \mathrm{~Hz}$. Respiration rate = average breaths per minute.

non-significant Group by Time interaction effect found, $F(6,72)=1.514, p=0.186, \eta^{2}=0.112$. Increases in total HRV - rest from Baseline to Week 1, Week 2, and Week 3 for the experimental group were more pronounced than for either the placebo or control groups.

Respiration rate - paced: There was a small non-significant Group by Time interaction effect found, F (5.23, $60.16)=0.566, p=0.733, \eta^{2}=0.047$. The groups essen- tially remained the same across time.

LF HRV - paced: There was a medium to large non-significant Group by Time interaction effect found, $F(6,66)$ $=1.478, p=0.200, \eta^{2}=0.118$. There was no clear evidence favouring the experimental group over the two control groups.

HF HRV - paced: There was a small non-significant Group by Time interaction effect found, $F(6,66)=0.265$, 
Table 3: Descriptive Statistics for Psychological Response to Sport Injury subscales at Baseline, Week 1, Week 2, and Week 3.

\begin{tabular}{|c|c|c|c|c|c|c|}
\hline \multirow{3}{*}{ Variable } & \multirow{3}{*}{ Group } & \multirow{3}{*}{$\mathbf{n}$} & \multicolumn{4}{|c|}{ Assessment } \\
\hline & & & Baseline & Week 1 & Week 2 & Week 3 \\
\hline & & & $\mathrm{M}(S D)$ & $M(S D)$ & $M(S D)$ & $\mathrm{M}(S D)$ \\
\hline \multirow[t]{3}{*}{ Devastation } & Experimental & 9 & $12.11(2.52)$ & $9.44(3.47)$ & $7.33(2.69)$ & $6.00(2.35)$ \\
\hline & Placebo & 9 & $7.22(3.42)$ & $8.11(4.83)$ & $7.44(3.36)$ & $7.56(4.22)$ \\
\hline & Control & 10 & $10.40(4.67)$ & $9.30(4.69)$ & $9.00(4.32)$ & $8.00(5.08)$ \\
\hline \multirow[t]{3}{*}{ Reorganization } & Experimental & 9 & $7.33(1.66)$ & $9.56(3.36)$ & $9.89(2.42)$ & $10.33(2.35)$ \\
\hline & Placebo & 9 & $9.67(2.40)$ & $10.44(3.09)$ & $10.56(3.05)$ & $10.67(2.50)$ \\
\hline & Control & 10 & $9.70(2.36)$ & $8.80(2.15)$ & $9.30(2.26)$ & $10.40(2.32)$ \\
\hline \multirow[t]{3}{*}{ Feeling Cheated } & Experimental & 9 & $9.44(3.05)$ & $8.11(2.76)$ & $7.33(2.40)$ & $6.67(2.74)$ \\
\hline & Placebo & 9 & $7.33(3.16)$ & $7.11(3.86)$ & $7.78(4.55)$ & $7.78(5.07)$ \\
\hline & Control & 10 & $8.60(4.20)$ & $9.20(4.85)$ & $9.10(4.98)$ & $8.50(5.19)$ \\
\hline \multirow[t]{3}{*}{ Restlessness } & Experimental & 9 & $9.78(2.28)$ & $8.33(2.35)$ & $7.44(1.81)$ & $5.78(2.22)$ \\
\hline & Placebo & 9 & $7.22(2.91)$ & $7.00(3.28)$ & $7.11(3.48)$ & $6.22(2.99)$ \\
\hline & Control & 10 & $9.20(3.46)$ & $9.60(5.19)$ & $9.00(4.90)$ & $8.40(5.48)$ \\
\hline \multirow[t]{3}{*}{ Isolation } & Experimental & 9 & $7.33(1.94)$ & $7.11(2.09)$ & $6.11(3.06)$ & $5.00(1.66)$ \\
\hline & Placebo & 9 & $5.33(1.66)$ & $5.22(1.56)$ & $6.11(2.76)$ & $5.44(2.30)$ \\
\hline & Control & 10 & $7.30(2.91)$ & $7.30(3.47)$ & $8.00(3.77)$ & $6.80(3.36)$ \\
\hline
\end{tabular}

Note: Psychological Response to Sport Injury subscales range from a low of 4 to a high of 20 , with the exception of reorganization, which ranges from a low of 3 to a high of 15 .

$p=0.951, \eta^{2}=0.024$. The groups essentially remained the same across time.

Total HRV - paced: There was a medium non-significant Group by Time interaction effect found, $F(6,66)=$ $0.699, p=0.651, \eta^{2}=0.060$. There was no clear evidence favouring the experimental group over the two control groups.

\section{Psychological Responses to Sport Injury}

Table 3 contains the group means and standard deviations for the psychological response to sport injury outcomes at Baseline, Week 1, Week 2, and Week 3.

Devastation: When controlling for baseline devastation scores, gender and injury history, a large non-significant Group by Time interaction effect in favour of the HRV experimental group was found, $F(4,44)=1.521, p=0.213$, $\eta^{2}=0.121$.

Reorganization: When controlling for baseline reorganization scores, a medium non-significant Group by Time interaction effect in favour of the HRV experimental group was found, $F(4,48)=0.798, p=0.532, \eta^{2}=$ 0.062 .

Isolation: A large significant Group by Time interaction effect in favour of the HRV experimental group was found, $F(5.53,69.07)=2.685, p=0.024, \eta^{2}=0.177$. Decreases in isolation from Baseline to Week 3 for the experimental group were significantly greater than for the placebo or control groups.

Restlessness: When controlling for injury history, a large non-significant Group by Time interaction effect in favour of the HRV experimental group was found, $F$ $(4.05,48.61)=1.804, p=0.143, \eta^{2}=0.131$.

Feeling cheated: When controlling for gender and injury history, a medium non-significant Group by Time in- teraction effect in favour of the HRV experimental group was found, $F(5.49,63.17)=1.112, p=0.365, \eta^{2}=0.088$.

\section{Pain Catastrophizing}

Table 4 contains the group means and standard deviations for the pain catastrophizing outcomes at Baseline, Week 1, Week 2, and Week 3.

Magnification: When controlling for injury history, a large non-significant Group by Time interaction effect in favour of the HRV experimental group was found, $F(6$, $72)=1.699, p=0.134, \eta^{2}=0.124$.

Rumination: When controlling for gender, a medium non-significant Group by Time interaction effect in favour of the HRV experimental group was found, $F(6,72)$ $=1.350, p=0.247, \eta^{2}=0.101$.

Helplessness: When controlling for injury history, a medium non-significant Group by Time interaction effect in favour of the HRV experimental group was found, $F(6,72)=0.886, p=0.509, \eta^{2}=0.069$.

\section{Relationships among variables}

Correlations between physiological change scores and psychological outcome change scores can be found in Table 5. Only respiration rate at rest showed significant relations with both psychological responses to injury and pain catastrophizing variables.

\section{Discussion}

The purpose of the study was to examine whether HRV biofeedback would improve athletes' pain catastrophizing and psychological responses following an injury. Overall, results indicate that injured athletes who received the HRV BFB intervention reported consistent medium to large improvements on all the psychological outcomes compared to their control counterparts. Be- 
Table 4: Descriptive Statistics for Pain Catastrophizing subscales at Baseline, Week 1, Week 2, and Week 3.

\begin{tabular}{|l|l|l|l|l|l|l|}
\hline Variable & Group & $\mathbf{n}$ & $\begin{array}{l}\text { Assessment } \\
\text { Baseline }\end{array}$ & Week 1 & Week 2 \\
M (SD) & Week 3 & (SD) & M (SD) \\
Rumination & Experimental & 9 & $8.22(4.24)$ & $5.67(3.87)$ & $5.78(3.73)$ & $3.89(3.92)$ \\
\hline & Placebo & 9 & $5.33(4.18)$ & $5.22(4.15)$ & $4.78(4.87)$ & $5.11(4.86)$ \\
\hline & Control & 10 & $8.90(4.07)$ & $7.30(4.76)$ & $4.50(4.30)$ & $6.20(4.54)$ \\
\hline Magnification & Experimental & 9 & $5.33(2.78)$ & $3.67(2.60)$ & $3.78(2.28)$ & $2.67(2.06)$ \\
\hline Helplessness & Placebo & 9 & $3.44(3.78)$ & $3.67(3.81)$ & $3.56(4.00)$ & $3.33(4.00)$ \\
& Control & 10 & $5.30(2.91)$ & $4.80(3.85)$ & $4.60(3.06)$ & $5.30(3.86)$ \\
\hline & Experimental & 9 & $8.22(3.80)$ & $4.44(2.51)$ & $5.00(4.18)$ & $3.56(4.85)$ \\
\hline & Placebo & 9 & $6.44(6.02)$ & $4.56(5.94)$ & $4.56(5.55)$ & $4.11(5.30)$ \\
\hline & Control & 10 & $8.00(4.47)$ & $6.70(5.50)$ & $5.00(3.97)$ & $5.70(6.08)$ \\
\hline
\end{tabular}

Note: Total pain catastrophizing scores range from a low of 0 to a high of 52; rumination subscale a low of 0 to a high of 16 ; magnification subscale a low of 0 to a high of 12; helplessness subscale a low of 0 to a high of 24 .

Table 5: Correlations between Physiological Change Scores and Psychological Outcome Change Scores.

\begin{tabular}{|c|c|c|c|c|c|c|c|c|}
\hline \multirow{2}{*}{$\begin{array}{l}\text { Psychological Responses } \\
\text { to Sport Injury }\end{array}$} & \multicolumn{8}{|c|}{ Physiological Outcomes } \\
\hline & $\begin{array}{l}\text { Respiration } \\
\text { Rate -Rest }^{\mathrm{a}}\end{array}$ & $\begin{array}{l}\text { LF HRV } \\
\text {-Rest }^{\mathrm{a}}\end{array}$ & $\begin{array}{l}\text { HF HRV } \\
\text {-Rest }^{a}\end{array}$ & $\begin{array}{l}\text { Total HRV } \\
\text {-Rest }\end{array}$ & $\begin{array}{l}\text { Respiration } \\
\text { Rate -Paced }\end{array}$ & $\begin{array}{l}\text { LF HRV } \\
\text {-Paced }^{b}\end{array}$ & $\begin{array}{l}\text { HF HRV } \\
\text {-Paced }^{b}\end{array}$ & $\begin{array}{l}\text { Total HRV } \\
\text {-Paced }^{\text {b }}\end{array}$ \\
\hline & $r$ & $r$ & $r$ & $r$ & $r$ & $r$ & $r$ & $r$ \\
\hline Devastation & $0.583^{* *}$ & 0.259 & 0.023 & 0.257 & 0.157 & -0.339 & -0.177 & -0.250 \\
\hline Reorganization & 0.339 & 0.000 & 0.114 & 0.054 & 0.198 & $-0.658^{* *}$ & -0.146 & $-0.471^{*}$ \\
\hline Feeling cheated & 0.350 & 0.149 & 0.112 & 0.247 & 0.065 & -0.188 & -0.101 & -0.042 \\
\hline Restlessness & $0.433^{*}$ & 0.126 & -0.129 & 0.085 & -0.144 & -0.132 & -0.159 & -0.054 \\
\hline Isolation & $0.499^{* *}$ & 0.300 & 0.131 & 0.359 & 0.224 & -0.071 & 0.137 & 0.058 \\
\hline \multicolumn{9}{|l|}{ Pain Catastrophizing } \\
\hline Rumination & $0.396^{*}$ & 0.218 & -0.140 & 0.136 & 0.186 & -0.008 & -0.348 & -0.093 \\
\hline Magnification & $0.424^{*}$ & 0.166 & -0.300 & -0.009 & 0.074 & -0.286 & -0.375 & -0.237 \\
\hline Helplessness & 0.163 & -0.040 & -0.358 & -0.148 & 0.271 & -0.214 & -0.375 & $-0.441^{*}$ \\
\hline
\end{tabular}

Note: ${ }^{a} \mathrm{n}=27 ;{ }^{\mathrm{b}} \mathrm{n}=25 ;{ }^{*} p<0.05,{ }^{* *} p<0.01$.

yond these general observations there are a number of specific findings that warrant commentary.

\section{Physiological indices}

With respect to the physiological indices associated with HRV BFB training, small to moderate non-significant group by time interaction effects were found for physiological indices during Task B - Paced Breathing Exercise. For the Task B HRV variables there was no clear pattern favoring a specific group. The respiratory rate data suggest that all participants were able to utilize the biofeedback information provided to match the rhythm of their breathing to a slowly oscillating respiratory pacer. However, during Task A - Rest period, when participants were left to their own devices and simply instructed to relax and breathe normally, significant and larger interaction effects emerged in favour of the intervention group for both respiration rate and LF HRV. Participants in the experimental group reported significantly greater increases in LF HRV at rest, suggesting HRV BFB training effectively increases LF HRV post-injury and throughout the rehabilitation process. The findings regarding LF HRV are consistent with those by Paul, et al. [24] who found that basketball players who underwent a HRV BFB intervention demonstrated significantly greater increases in LF HRV from pre to post-intervention, than those in either the placebo or control groups.
This change in LF HRV is proposed to be due to individuals reducing their respiratory rate and breathing in a slow and controlled manner causing respiratory sinus arrhythmia to shift from the HF to the LF range of HRV $[27,39]$. Secondly, while respiration rates during Task A (rest period) remained similar for those in the placebo and control groups; the experimental group displayed significant reductions in respiration rate at each week, compared to Baseline. Overall, the experimental group in the present study had significantly lower respiration rates than injured athletes who were not provided with HRV BFB training. Paul, et al. [24] also found respiration rates to be significantly reduced in basketball players who received HRV BFB training compared to the control group. Thus, HRV BFB may be used to enhance individuals' awareness and control over their respiratory patterns to aid in self-regulation and relaxation. The connection between self-awareness and respiration relates to the reciprocal relationship noted in the biopsychosocial model [23] and holds promise for practical application in rehabilitation as a tool for athletes to assist in self-regulation strategies.

The group by time interaction effects for total HRV and HF HRV at rest were moderate in size and failed to reach significance. Our finding concerning HF HRV is consistent with previous research, which has found no significant differences in HF HRV at baseline and fol- 
low-up sessions of treatment $[27,40]$. Wheat and Larkin [39] conducted a critical review on the effectiveness of HRV and related biofeedback across 14 studies to improve HRV outcomes and reported that all results are in agreement that HF HRV does not significantly increase in consequent to HRV BFB treatment. It has been suggested that as respiratory sinus arrhythmia shifts from the high frequency to the low frequency range of HRV during slow, paced breathing involved in BFB training, increases in HF HRV are unexpected [39]. Although the interaction effect for total HRV was non-significant, group means suggest that increases for those receiving HRV BFB were more pronounced than for either the placebo or control groups. This finding is consistent with previous research, which has found evidence for longterm changes in total HRV to be tenuous [39]. Effects of HRV BFB training on total HRV in injured athlete's warrants further attention.

\section{Psychological responses to sport injury}

For psychological responses to sport injury group differences over time were non-significant, however, the effects were medium to large in size and consistently favoured the experimental group. This suggests that reductions in devastation, feeling cheated, and restlessness across time were more pronounced for individuals in the experimental group compared to those in the placebo or control groups. Only isolation reached significance with a large effect in favour of the experimental group, indicating individuals in the experimental group reported significantly greater reductions in feelings of isolation over time. In terms of reorganization, group differences over time also failed to reach significance; however, the effect was medium in size and favoured the experimental group. This suggests that increases in feelings of reorganization across time were more pronounced for those in the experimental group than for those in either the placebo or control groups. Taken together, there is consistent evidence that HRV BFB training positively influenced psychological responses throughout the rehabilitation process following injury for the current sample. Nevertheless, these findings need to be replicated using a larger sample size before definitive conclusions can be drawn. These findings are in line with those of Evans and Hardy [16] who investigated the use of goal setting as a form of psychological-based intervention for injured athletes. Whereas Evans and Hardy [16] only reported significant effects for time with respect to psychological responses to sport injury, this pilot study demonstrated medium-to-large, albeit non-significant interaction effects for a number of these responses in favour of the experimental group. Our findings are also congruent with previous research, which has indicated the beneficial effects of HRV BFB training on stress, anxiety, negative effect, and mood disturbances in sport and clinical settings alike $[25,27,28,40]$.

Researchers have proposed a number of alternative mechanisms through which HRV BFB could yield positive psychological outcomes. Paul and Garg [28] suggested that HRV BFB training is a self-regulatory intervention, which may contribute to reductions in psychophysiological stressors (e.g. anxiety/heart rate, arousal/vasodilation) and support optimal performance. Further, Hassett, et al. [40] suggested that experiences of relaxation, stress reduction, and the chance to become an active participant in treatment can enhance the patient's perceived level of control and self-esteem. Finally, Karavidas, et al. [27] proposed that HRV training may include symptom amelioration through increased attention to one's breathing patterns and increased self-efficacy in self-regulation of mood states.

\section{Pain catastrophizing}

With regards to pain catastrophizing, group differences over time were non-significant, however, the effects were moderate in size and consistently favoured the experimental group. These findings suggest that HRV BFB training may contribute to declines in pain magnification, rumination, and helplessness in injured athletes post-injury and throughout the rehabilitation process. These findings are in line with previous research by Riddle, et al. [41], who investigated the effects of a pain coping skills intervention in patients scheduled for knee arthroplasty procedures, and found those who received coping skills training demonstrated significantly greater reductions in pain catastrophizing, compared to a usual care cohort. Other investigations in recent years have demonstrated the role catastrophizing may play in the rehabilitation process $[9,13,14]$, as well as recommended incorporating guidelines for reducing emotional disturbance and enhancing the rehabilitation experience [9]. Catastrophizing has been shown to impact perceptions of severity, distress, poor treatment outcomes $[13,14]$, and is associated with not only experiencing a greater amount of pain, but for a longer period of time [42]. As well, individuals with more severe injuries have exhibited the use of more positive coping strategies than those with less severe injuries [9]. Results of this line of inquiry provide support for the use of BFB as a potentially useful and efficacious tool in rehabilitation.

\section{Relationships among outcomes}

A number of change score correlations were found between physiological indices and psychological outcomes. Significant, positive associations were found between change in respiration rate - rest and changes in rumination, magnification, devastation, restlessness, and isolation. Given the large training effect on respiration rate at rest found for the intervention group, and the large effects found favoring the intervention group on the abovementioned psychological outcomes, it is not surprising that these consistent relationships were found. The direction and magnitude of these change score correlations provide support for the proposed physiological mechanism through which HRV BFB may 
lead to beneficial psychological outcomes. Perhaps we might have found stronger psychological outcome effects if the association between LF HRV - rest and the psychological variables of interest were more robust. The large effect favoring the intervention observed for LF HRV suggests that it had the potential to produce a psychological response to injury effect. A few significant, negative correlations emerged between Task B - paced HRV variables and psychological outcomes. Although this is not what was expected, it is not unusual given how paced HRV indices operated across time.

\section{Strengths and Limitations}

There are several strengths associated with the present pilot study. As an intervention study exploring the effects of a novel and new approach, the inclusion of both a placebo and a control group was a relative strength. Prior research regarding the use of HRV BFB in other populations and other psychological-based interventions within the athletic injury setting have emphasized the importance of utilizing a control and/or placebo group in study designs [39,43]. Cupal [43] argued that without adequate controls in psychological interventions, it is difficult to separate treatment effects from effects of other intervening variables. Strength was the implementation of a pre-existing HRV BFB training protocol outlined by Lehrer, et al. [36] that has been used in the majority of previous studies employing HRV BFB interventions, which increased the efficacy and procedural reliability in the current study. Furthermore, inclusion of multiple assessments allowed examination over the duration of a three-week period, rather than just at baseline and post-intervention. Given that previous research supports the idea that negative effect, mood disturbance, and psychological distress tend to dissipate over time $[7,44]$, multiple assessment periods allowed the researchers to examine if psychological responses of athletes differed across time depending on the specific training, or lack thereof received.

Despite these strengths, there are a number of limitations that must be acknowledged with this work. The characteristics of the sample studied must be noted and understood in the interpretation of study findings, as well as for future study recommendations. Group differences were found for gender and injury history. In addition, these were both treated as covariates in the primary analyses as they were significantly related with several primary psychological outcomes at baseline. Future studies should consider screening for injury history during recruitment in order to examine the extent of athletes' psychological response having never been through a rehabilitation process before. Although recruitment was preferred immediately post-injury, the majority of participants were recruited more than a week after sustaining the injury. Future studies should also consider screening for time since injury, given aforementioned affect and mood disturbance changes across time $[7,44]$. Although several injury-related factors were measured and controlled for in this pilot RCT, unfortunately whether injured athletes had received or were scheduled to receive surgery for their injury was not formally assessed. Furthermore, a larger sample size would have served to increase statistical power, especially for those measures bordering significance for an interaction effect. Finally, as the purpose of this study was to examine the effectiveness of a HRV BFB intervention (i.e., supervised HRV BFB training sessions and recommendations to practice learned techniques daily), it should be acknowledged that this study was unable to disentangle the contribution of the supervised HRV BFB training sessions versus home-based practice on the respective primary outcomes.

\section{Future Directions}

Future investigators are encouraged to design and implement $B F B$ interventions for injured athletes. Wheat and Larkin [39] emphasized the importance of replicating BFB investigations; because until studies have been embedded within a larger network of investigations surrounding HRV BFB's effects in the same population, each study completed continues to be isolated and a complete understanding will not be reached. Investigation is warranted of the potential mechanisms by which HRV BFB may lead to beneficial outcomes. Until this occurs, the most effective BFB interventions cannot be implemented and psychological responses will not be fully understood. Additionally, exploring the effects of combining HRV BFB with more traditional psychological-based intervention techniques could prove to be a viable avenue. Paul and Garg [28] suggested combining HRV BFB with relaxation imagery, progressive muscle relaxation, and other forms of psychological-based interventions so as to generate physiological and psychological domains more consistently and extensively. It would also be interesting for future studies to compare the effectiveness of HRV BFB versus other psychological-based interventions such as relaxation training, which may be more portable and less expensive options for managing psychological responses of injured athletes. Given the established relationship between pain catastrophizing and adverse recovery outcomes, perhaps future intervention studies should consider screening for and including only individuals who meet the clinical threshold for pain catastrophizing [45] so as to examine the potential viability of BFB treatment for pain related fear more extensively. In our current sample 16 out of the 28 participants exceeded this pain threshold. Furthermore, there is a strong call for future investigations employing the use of HRV BFB in athletic injury to investigate its potential effects on recovery outcomes, return to play status, and satisfaction with rehabilitation. Inclusion of qualitative methods to explore athletes' perceptions of HRV BFB as a psychological intervention is recommended. Continued efforts are also needed to validate methods of measuring and analyzing HRV and to strengthen protocols for HRV BFB training. 


\section{Conclusion}

The present pilot study provides preliminary evidence that HRV BFB training is associated with improvements in pain catastrophizing (e.g., rumination) and the psychological responses (e.g., devastation) of injured athletes throughout the rehabilitation process. Further, BFB training is associated with a number of salient physiological indices (i.e., lower respiration rate at rest and higher LF HRV at rest). Given the considerable evidence indicating that pain catastrophizing contributes to poor recovery outcomes and lack of improvement [15], and that negative psychological responses are associated with not returning to a pre-injury level of sport following injury [46], it is possible that HRV BFB may be an effective strategy to improve these outcomes and increase the likelihood of a successful return to sport. This evidence may inform the development of future HRV BFB interventions, and provides support for the inclusion of HRV BFB as a viable psychological-based intervention to improve psychological responses and pain catastrophizing throughout the rehabilitation process. A large and adequately powered randomized controlled trial is warranted.

\section{References}

1. Statistics Canada. Canadian Community Health Survey: Combined data, 2013/2014. (Catalogue number 82-625-X). Statistics Canada Website.

2. Bauer R, Steiner M (2009) Injuries in the European Union statistics summary 2005-2007. Vienna: Eurosafe and KfV.

3. National Safety Council (2015) Injury Facts ${ }^{\circledR}, 2015$ edition. Itasca, IL.

4. Evans L, Wadey R, Hanton S, Mitchell I (2012) Stressors experienced by injured athletes. J Sports Sci 30: 917-927.

5. Salim J, Wadey R, Diss C (2015) Examining the relationship between hardiness and perceived stress-related growth in a sport injury context. Psychol Sport Exerc 19: 10-17.

6. Wiese-Bjornstal DM, Smith AM, Shaffer SM, Morrey MA (1998) An integrated model of response to sport injury: psychological and sociological dynamics. J Appl Sport Psychol 10: 46-69.

7. Tracey J (2003) The emotional response to the injury and rehabilitation process. J Appl Sport Psychol 15: 279-293.

8. Johnston LH, Carroll D (1998) The context of emotional responses to athletic injury: A qualitative analysis. J Sport Rehabil 7: 206-220.

9. Masten R, Strazar K, Zilavec I, Tusak M, Kandare M (2014) Psychological response of athletes to injury. Kinesiology 46: 127-134.

10. Ardern CL, Taylor NF, Feller JA, Webster KE (2013) A systematic review of the psychological factors associated with returning to sport following injury. $\mathrm{Br} J$ Sports Med 47: 11201126.

11. Leung $L$ (2012) Pain catastrophizing: an updated review. Indian J Psychol Med 34: 204-217.

12. Sullivan MJ, Bishop S, Pivik J (1995) The pain catastrophizing scale: Development and validation. Psychol Assessment 7: 524-532.

13. de Boer MJ, Struys MM, Versteegen GJ (2012) Pain-related catastrophizing in pain patients and people with pain in the general population. Eur J Pain 16: 1044-1052.

14. Slepian P, Bernier E, Scott W, Niederstrasser NG, Wideman T, et al. (2014) Changes in pain catastrophizing following physical therapy for musculoskeletal injury: the influence of depressive and post-traumatic stress symptoms. J Occup Rehabil 24: 22-31.

15. Bergbom S, Boersma K, Overmeer T, Linton SJ (2011) Relationship among pain catastrophizing, depressed mood, and outcomes across physical therapy treatments. Phys Ther 91: 754-764.

16. Evans L, Hardy L (2002) Injury rehabilitation: A goal-setting intervention study. Res Q Exerc Sport 73: 310-319.

17. Cupal DD, Brewer BW (2001) Effects of relaxation and guided imagery on knee strength, reinjury anxiety, and pain following anterior cruciate ligament reconstruction. Rehabil Psychol 46: 28-43.

18. Ross MJ, Berger RS (1996) Effects of stress inoculation training on athletes' postsurgical pain and rehabilitation after orthopedic injury. J Consult Clin Psychol 64: 406-410.

19. Maddison R, Prapavessis H, Clatworthy M (2006) Modeling and rehabilitation following anterior cruciate ligament reconstruction. Ann Behav Med 31: 89-98.

20. Wesch N, Hall C, Prapavessis H, Maddison R, Bassett S, et al. (2012) Self-efficacy, imagery use, and adherence during injury rehabilitation. Scand J Med Sci Sports 22: 695-703.

21. Hanin Y, Hanina M (2009) Optimization of performance in top-level athletes: An action-focused coping approach. Int J Sports Sci Coach 4: 47-91.

22. Levitt R, Deisinger JA, Remondet Wall J, Ford L, Cassisi JE (1995) EMG feedback-assisted postoperative rehabilitation of minor arthroscopic knee surgeries. J Sports Med Phys Fitness 35: 218-223.

23. Brewer BW, Andersen MB, Van Raalte JL (2002) Psychological aspects of sport injury rehabilitation: Toward a biopsychological approach. In: Mostofsky DL, ZaichowskyLD, Medical and Psychological Aspects of Sport and Exercise. Morgantown, WV: FIT, 41-54.

24. Paul M, Garg K, Singh Sandhu J (2012) Role of biofeedback in optimizing psychomotor performance in sports. Asian J Sports Med 3: 29-40.

25. Lagos L, Vaschillo $E$, Vaschillo $B$, Lehrer $P$, Bates $M$, et al. (2008) Heart rate variability biofeedback as a strategy for dealing with competitive anxiety: A case study. Biofeedback 36: 109-115.

26. Aubert AE, Seps B, Beckers F (2003) Heart rate variability in athletes. Sports Med 33: 889-919.

27. Karavidas MK, Lehrer PM, Vaschillo E, Vaschillo B, Marin $\mathrm{H}$, et al. (2007) Preliminary results of an open label study of heart rate variability biofeedback for the treatment of major depression. Appl Psychophysiol Biofeedback 32: 19-30.

28. Paul M, Garg K (2012) The effect of heart rate variability biofeedback on performance psychology of basketball players. Appl Psychophysiol Biofeedback 37: 131-144.

29. Appelhans BM, Luecken LJ (2006) Heart rate variability as an index of regulated emotional responding. Rev Gen Psychol 10: 229-240.

30. Olivier N, Legrand R, Rogez J, Gamelin FX, Berthoin S, et al. (2007) Heart rate variability before and after knee surgery in amateur soccer players. J Sport Rehabil 16: 336342. 
31. Lehrer PM, Gevirtz R (2014) Heart rate variability biofeedback: how and why does it work? Front Psychol 5: 756.

32. Hallman DM, Olsson EM, von Schéele B, Melin L, Lyskov $E$ (2011) Effects of heart rate variability biofeedback in subjects with stress-related chronic neck pain: a pilot study. Appl Psychophysiol Biofeedback 36: 71-80.

33. Draper V, Ballard L (1991) Electrical stimulation versus electromyographic biofeedback in the recovery of quadriceps femoris muscle function following anterior cruciate ligament surgery. Phys Ther 71: 455-461.

34. Thabane L, Ma J, Chu R, Cheng J, Ismaila A, et al. (2010) A tutorial on pilot studies: the what, why and how. BMC Med Res Methodol 10: 1.

35. Fuller CW, Ekstrand J, Junge A, Andersen TE, Bahr R, et al. (2006) Consensus statement on injury definitions and data collection procedures in studies of football (soccer) injuries. Scand J Med Sci Sports 16: 83-92.

36. Lehrer PM, Vaschillo EG, Vaschillo B (2000) Resonant frequency biofeedback training to increase cardiac variability: Rationale and manual for training. Appl Psychophysiol Biofeedback 25: 177-191.

37. Evans L, Hardy L, Mitchell I, Rees T (2008) The development of a measure of psychological responses to injury. $J$ Sport Rehabil 17: 21-37.

38. Stevens J (1996) Applied Multivariate Statistics for the Social Sciences. Mahway, NJ: Lawrence Erlbaum Associates.

39. Wheat AL, Larkin KT (2010) Biofeedback of heart rate variability and related physiology: a critical review. Appl Psychophysiol Biofeedback 35: 229-242.
40. Hassett AL, Radvanski DC, Vaschillo EG, Vaschillo B, Sigal $\mathrm{LH}$, et al. (2007) A pilot study of the efficacy of heart rate variability (HRV) biofeedback in patients with fibromyalgia. Appl Psychophysiol Biofeedback 32: 1-10.

41. Riddle DL, Keefe FJ, Nay WT, McKee D, Attarian DE, et al. (2011) Pain coping skills training for patients with elevated pain catastrophizing who are scheduled for knee arthroplasty: A quasi-experimental study. Arch Phys Med Rehabil 92: 859-865.

42. Pavlin DJ, Sullivan MJ, Freund PR, Roesen K (2005) Catastrophizing: a risk factor for postsurgical pain. Clin $\mathrm{J}$ Pain 21: 83-90.

43. Cupal DD (1998) Psychological interventions in sport injury prevention and rehabilitation. J Appl Sport Psychol 10: 103123.

44. Brewer BW, Cornelius AE, Sklar JH, Van Raalte JL, Tennen $\mathrm{H}$, et al. (2007) Pain and negative mood during rehabilitation after anterior cruciate ligament reconstruction: A daily process analysis. Scand J Med Sci Sports 17: 520-529.

45. Sullivan MJ, Adams H, Rhodenizer T, Stanish WD (2006) A psychosocial risk factor--targeted intervention for the prevention of chronic pain and disability following whiplash injury. Phys Ther 86: 8-18.

46. Ardern CL, Taylor NF, Feller JA, Whitehead TS, Webster KE (2015) Sports participation 2 years after anterior cruciate ligament reconstruction in athletes who had not returned to sport at 1 year: A prospective follow-up of physical function and psychological factors in 122 athletes. Am J Sports Med 43: 848-856. 